\title{
SEGMENTING NON STATIONARY IMAGES WITH TRIPLET MARKOV FIELDS
}

\author{
Dalila Benboudjema and Wojciech Pieczynski \\ INT/GET, Département CITI, CNRS UMR 5157 \\ 9, rue Charles Fourier, 91000 Evry, France \\ tél. 01607644 52, fax 01607644 33, E-mail Dalila.Benboudjema@int-evry.fr \\ tél. 01607644 25, fax 01607644 33, E-mail Wojciech.Pieczynski@int-evry
}

\begin{abstract}
The hidden Markov field (HMF) model has been used in many model-based solutions to image analysis problems, including that of image segmentation, and generally gives satisfying results. However, when the class image is non stationary, the unsupervised segmentation results provided by HMF can be poor. In this paper, we tackle the problem of modeling a non stationary hidden random field and its effect on the unsupervised statistical image segmentation. We propose an original approach, based on the recent triplet Markov field (TMF) model, to segment non stationary images. Experiments indicate that the new algorithm performs better than the classical one.
\end{abstract}

\section{INTRODUCTION}

Image segmentation is a fundamental and yet difficult task in machine vision. Among several models and approaches developed, hidden Markov fields (HMF) and Bayesian segmentation based on them, can be of outstanding efficiency in numerous situations. Hundreds papers have been written on the subject since the seminal articles $[2,4,5,7]$ and a rich bibliography can be seen in [10]. However, when considering non stationary images, which will be in this paper class images, and unsupervised segmentation, HMF model is difficult to apply. In fact, to model non stationarity of the class image one has to make depend the form potential functions (defining the Markovian distribution of the class field) on pixels. Now, such dependence makes very difficult the estimation of the model parameters when considering the unsupervised segmentation framework (in which HMF parameters are estimated from the observed image). The idea of this paper is to use a third random field whose realizations would model the simultaneous presence of different stationarities in the class image.
Let us notice that similar general ideas concerning the hidden Markov chains (HMC) and triplet Markov chains (TMC) are presented in [6]; however, models and processing considered here are completely different.

\section{HIDDEN AND TRIPLET MARKOV FIELDS}

Let $S$ be the set of pixels. HMF model contains two stochastic processes $X=\left(X_{s}\right)_{s \in S}$ and $Y=\left(Y_{s}\right)_{s \in S}$, in which $X=x$ is unobservable - or hidden - and has to be recovered from the observed $Y=y$. Therefore, $Y=y$ can be seen as a noisy version of $X=x$. In this paper we consider the problem of digital image segmentation : each $X_{s}$ takes its values in a finite set of classes $\Omega=\left\{\omega_{1}, \ldots, \omega_{k}\right\}$, whereas each $Y_{s}$ takes its values in the set of real numbers $R$. The distribution $p(x)$ of the field $X$ is a Markov distribution, and it is the same with its posterior distribution $p(x \mid y)$, which is its distribution conditional on $Y=y$. The Markovianity of $p(x \mid y)$ is crucial because it enables one to estimate the marginals $p\left(x_{s} \mid y\right)$, and thus enables one to apply the Bayesian Maximum Posterior Mode (MPM) segmentation method [7]. Albeit this model has been thoroughly tested in the stationary case, it can fail in the non stationary one, in which the parameters defining the potential functions on cliques can vary when cliques vary in the set of pixels.

The aim of this paper is to present a novel approach that enables one to overcome this problem. Our contributions are twofold. First, the proposed approach consists of using the recent "Triplet" Markov fields (TMF [8]) model, in which one introduces a third random field $U=\left(U_{s}\right)_{s \in S}$, each $U_{s}$ taking its values in a finite set $\Lambda=\left\{\lambda_{1}, \ldots, \lambda_{M}\right\}$. The idea is to interpret the $M$ possible values of $U_{s}$ as $M$ different possible stationarities of 
$X=x$. The triplet $T=(X, U, Y)$ being assumed Markovian, $p\left(x_{s}, u_{s} \mid y\right)$ is computable for the same reasons as above, and thus $p\left(x_{s} \mid y\right)$ is given by $p\left(x_{s} \mid y\right)=\sum_{u_{s} \in \Lambda} p\left(x_{s}, u_{s} \mid y\right)$. Having $p\left(x_{s} \mid y\right)$ enables us to perform MPM segmentation as in classical HMF.

The second novelty of this paper is to adapt the parameter estimation method described in [1], based on the general "Iterative Conditional Estimation" (ICE [3, 9]) to the particular TMF considered in this paper.

Finally, we show, via experiments, that unsupervised segmentation methods based on ICE and TMF can be significantly more efficient that those based on ICE and HMF.

Let us consider, as an example, the distribution of the Markov field $X=\left(X_{s}\right)_{s \in S}$ defined by the energy

$$
W(x)=\sum_{(s, t) \in C_{H}} \alpha_{H}\left(1-2 \delta\left(x_{s}, x_{t}\right)\right)+\sum_{(s, t) \in C_{V}} \alpha_{V}\left(1-2 \delta\left(x_{s}, x_{t}\right)\right)(1)
$$

Where $C_{H}$ is the set of couples of pixels horizontally neighbours, $C_{V}$ is the set of couples of pixels vertically neighbours, and $\delta\left(x_{s}, x_{t}\right)$ verifies $\delta\left(x_{s}, x_{t}\right)=1$ for $x_{s}=x_{t}$, and $\delta\left(x_{s}, x_{t}\right)=0$ for $x_{s} \neq x_{t}$. The field $X=\left(X_{s}\right)_{s \in S}$ is then classically Markovian with respect to four nearest neighbours.

Furthermore, let us consider classically that the random variables $\left(Y_{s}\right)_{s \in S}$ are independent conditionally on $X=\left(X_{s}\right)_{s \in S}$. The distribution of $(X, Y)$ is then written

$$
p(x, y)=\gamma \exp \left[-W(x)+\sum_{s \in S} \log \left(p\left(y_{s} \mid x_{s}\right)\right)\right]
$$

We generalize this model by considering $(X, U)$, with two stationarities $\Lambda=\{a, b\}$, whose distribution is defined by the energy

$$
\begin{aligned}
& W(x, u)=\sum_{(s, t) \in C_{H}} \alpha_{H}^{1}\left(1-2 \delta\left(x_{s}, x_{t}\right)\right)- \\
& \left(\alpha_{a H}^{2} \delta *\left(u_{s}, u_{t}, a\right)+\alpha_{b H}^{2} \delta *\left(u_{s}, u_{t}, b\right)\right)\left(1-\delta\left(x_{s}, x_{t}\right)\right) \\
& +\sum_{\left(s, t \in C_{V}\right.} \alpha_{V}^{1}\left(1-2 \delta\left(x_{s}, x_{t}\right)\right)- \\
& \left(\alpha_{a V}^{2} \delta *\left(u_{s}, u_{t}, a\right)+\alpha_{b V}^{2} \delta *\left(u_{s}, u_{t}, b\right)\right)\left(1-\delta\left(x_{s}, x_{t}\right)\right)
\end{aligned}
$$

With $\quad \delta *\left(u_{s}, u_{t}, a\right)=1 \quad$ for $\quad u_{s}=u_{t}=a, \quad$ and $\delta *\left(u_{s}, u_{t}, a\right)=0 \quad$ otherwise and $\delta\left(u_{s}, u_{t}, b\right)=1$ for $u_{s}=u_{t}=a$, and $\delta *\left(u_{s}, u_{t}, b\right)=0$ otherwise. We can easily verify that for $x=u$ the energy (3) is reduced to the energy (1); therefore the model (3) is a generalisation of the classical model (1).

Furthermore, let us define the distribution of $(X, U, Y)$ by

$$
p(x, u, y)=\gamma \exp \left[-W(x, u)+\sum_{s \in S} \log \left(p\left(y_{s} \mid x_{s}\right)\right)\right]
$$

Both models HMF given by (2) and TMF given by (4) allows one to estimate $p\left(x_{s} \mid y\right)$. In HMF this is classically done from (2) using the Gibbs sampler, and in TMF this is done in two steps : (i) estimate $p\left(x_{s}, u_{s} \mid y\right)$ by the Gibbs sampler; (ii) calculate $p\left(x_{s} \mid y\right)=\sum_{u_{s} \in \Lambda} p\left(x_{s}, u_{s} \mid y\right)$. Therefore, the Bayesian Maximum Posterior Mode (MPM) can be used in both HMF and TMF given by (2) and (4), respectively.

\section{EXPERIMENTS}

We give below four examples of the use of the TMF model given by (4) in unsupervised image segmentation. As specified above, all parameters are estimated from $Y=y$ by a particular algorithm belonging to the so called "ICE" family of methods [1].

The two first examples concern simulations with respect to the new model (4). In each of the two cases considered, $(X, U)$ is first simulated according to (3), and then $X=x$ is corrupted with Gaussian white noise with means $m_{1}=0 ., \quad m_{2}=2 ., \quad$ and variances $\sigma_{1}^{2}=\sigma_{2}^{2}=1$. Fig. 1 corresponds to $\alpha_{H}^{1}=\alpha_{V}^{1}=1$., $\alpha_{a H}^{2}=\alpha_{a V}^{2}=0.5, \quad \alpha_{b H}^{2}=\alpha_{b V}^{2}=-0.4$, whereas Fig.2 corresponds to $\alpha_{H}^{1}=\alpha_{V}^{1}=1 ., \quad \alpha_{a H}^{2}=1 ., \quad \alpha_{a V}^{2}=-0.3$, $\alpha_{b H}^{2}=-0.3, \quad \alpha_{b V}^{2}=1$. The realizations of $U$ corresponding to these two cases are given in Fig. 3. We can see that results in Fig. 2 are particularly striking. The third example concerns a hand-drawn image $x$, noisy as above with $m_{1}=0 ., \quad m_{2}=1.6, \quad$ and $\sigma_{1}^{2}=\sigma_{2}^{2}=1$. Such an image is neither TMF nor HMF and we can see, according to results in Fig. 4, that the new method is of interest.

The fourth example concerns an application of the proposed model to a real image. Fig. 5 compares results obtained with the new TMF model based unsupervised segmentation and the classical HMF based one. This 430x430 radar image represents a part of the Netherlands and contains four classes. As we have no ground truth, it is difficult to draw rigorous conclusions; 
however, in some spots we better recover some details like connections between canals, which are represented by the black lines in the image.

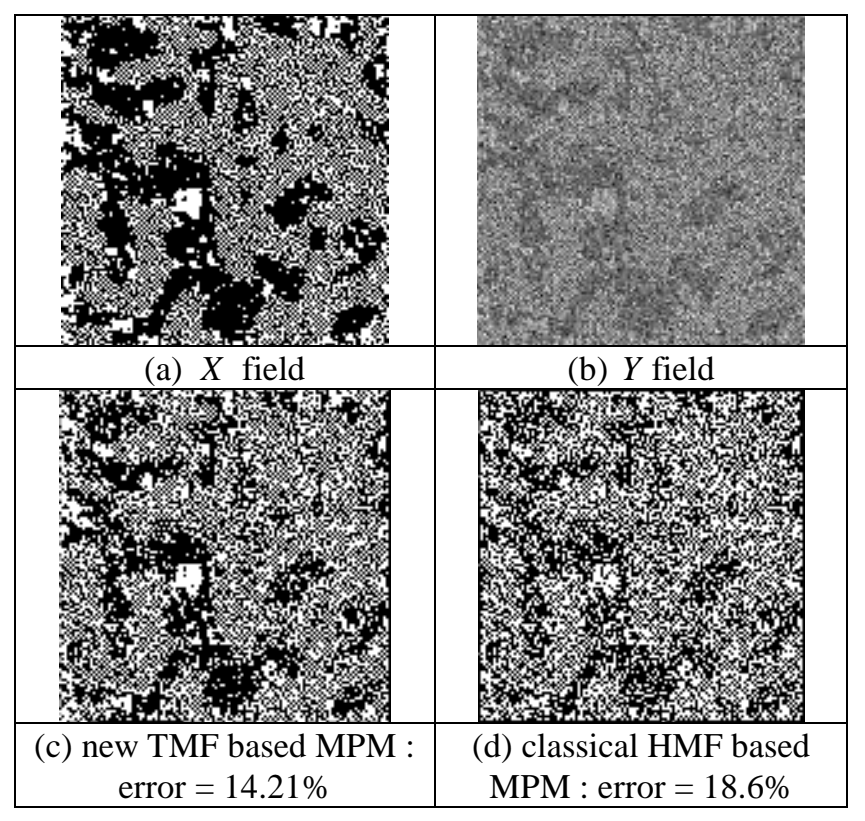

Fig. 1 : Simulated images using the new TMF model (a, b) and two MPM based on TMF and classical HMF unsupervised segmentation results $(\mathrm{c}, \mathrm{d})$.

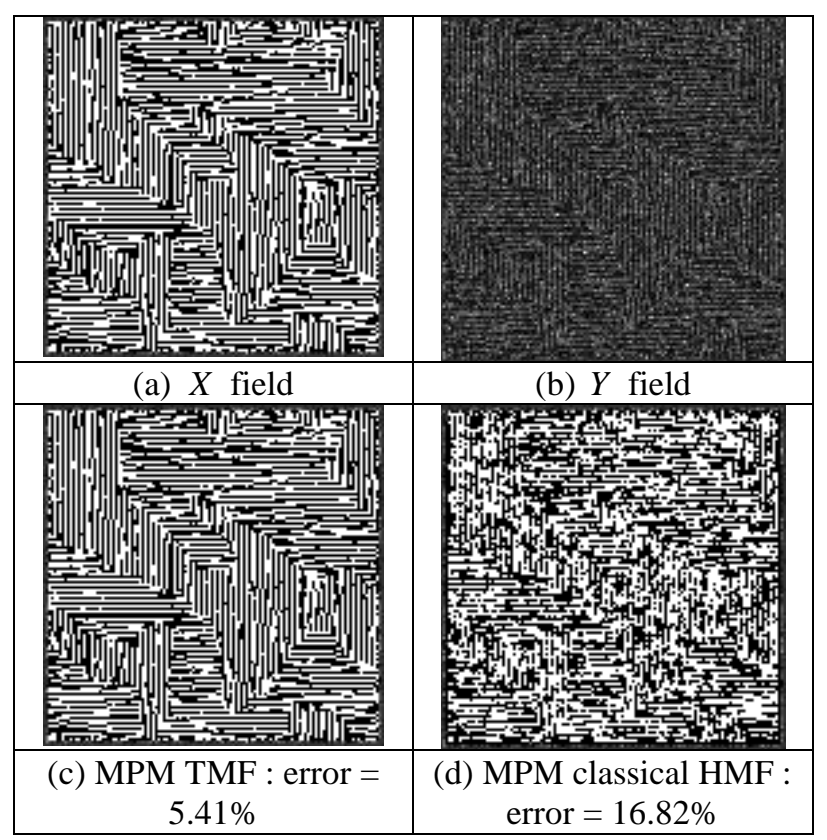

Fig. 2 : Simulated images using the new TMF model and two MPM based on TMF and classical HMF unsupervised segmentation results.

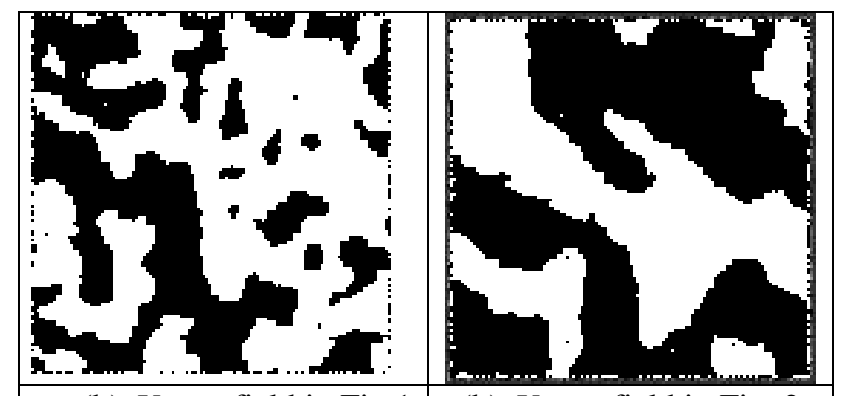

(b) $U=u$ field in Fig.1

(b) $U=u$ field in Fig. 2

Fig. 3 : Realizations of $U$ in Fig. 1 and Fig. 2.

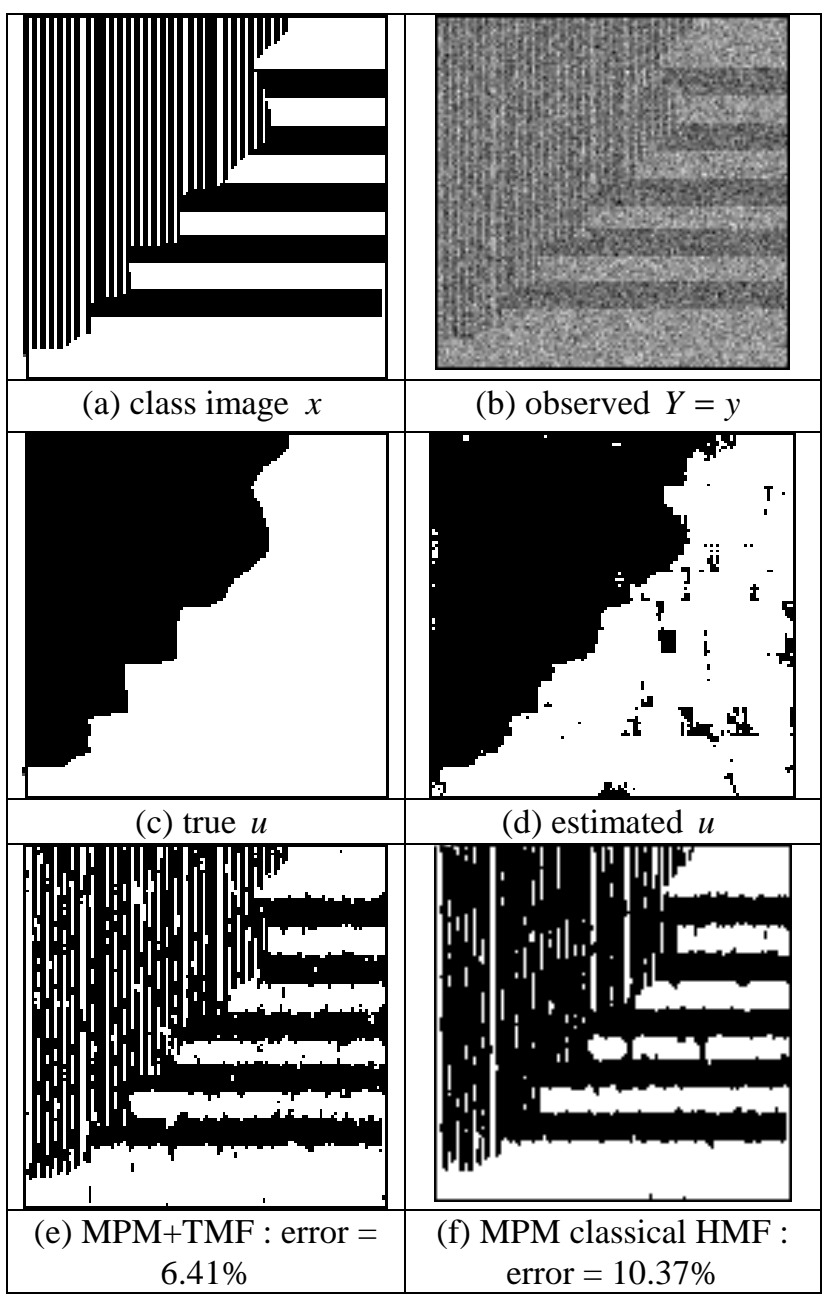

Fig. 4 : Hand-drawn class image $x$ (a), its noisy version (b), $u$ corresponding to $x$ (c), estimated $u$ (d), new method unsupervised segmentation result (e), and classical method one (f). 


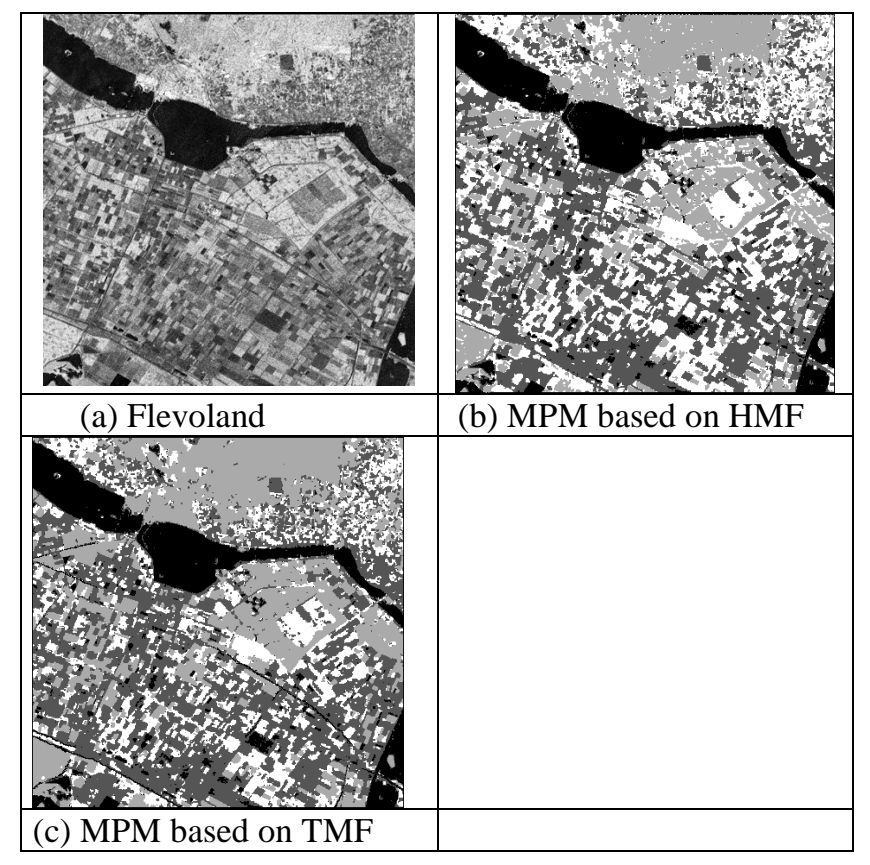

Fig. 5 : Real image segmentation (four classes) with the new method and a classical one

All the segmented images presented above have been obtained after 100 MPM samples, each of which is performed by 20 Gibbs sampler iterations.

Let us remark that we focused in this paper on recovering $X=x$ from $Y=y$; however, $U=u$ can also have a physical interpretation and its estimation can be of interest. For example, let us imagine that there are two classes "houses" and "trees". Otherwise, a half of the image $X=x$ is a "town", and the other half is "outside town". Furthermore, let us assume that there are trees in town, and there are houses outside town. As the distribution of $X$ is different in "town" and "outside town", these two possibilities will be model, according to our model, by two stationarities $\lambda_{1}, \lambda_{2}$. Therefore, for a given pixel $s \in S$, we can say that $x_{s}$ is "house" or "trees", and $u_{s}$ is 'town" or "outside town", and both of them are of interest. As $p\left(u_{s} \mid y\right)$ is computable from $p\left(x_{s}, u_{s} \mid y\right)$ by $p\left(u_{s} \mid y\right)=\sum_{x_{s} \in \Omega} p\left(x_{s}, u_{s} \mid y\right), U=u$ can be estimated from $Y=y$ by MPM. One such example is provided in Fig. 4.

\section{CONCLUSION}

We dealt in this with unsupervised Bayesian segmentation of non stationary images. We showed : (i) the recent TMF model [8] can model non stationarity in an efficient way; (ii) TMF based unsupervised segmentation of non stationary images can be more efficient than the classical HMF based one.

\section{REFERENCES}

[1] D. Benboudjema and W. Pieczynski, 'Parameter estimation in pairwise Markov fields", Advanced Concepts for Intelligent Vision Systems (ACVIS 04), Aug. 31-Sept. 3, Brussels, Belgium, 2004.

[2] J. Besag, "On the statistical analysis of dirty pictures", Journal of the Royal Statistical Society, Series B, 48, pp. 259-302, 1986.

[3] J.-P. Delmas, "An equivalence of the EM and ICE algorithm for exponential family", IEEE Trans. on Signal Processing, Vol. 45, No. 10, pp. 2613-2615, 1997.

[4] H. Derin and H. Elliott, "Modeling and Segmentation of Noisy and textured images using Gibbs random fields," IEEE Trans. on Pattern Analysis and Machine Intelligence, Vol. 9, No. 1, pp. 39-55, 1987.

[5] S. Geman and D. Geman, 'Stochastic relaxation, Gibbs distributions and the Bayesian restoration of images," IEEE Trans. on Pattern Analysis and Machine Intelligence, Vol. 6, pp. 721-741, 1984.

[6] P. Lanchantin and W. Pieczynski, 'Unsupervised non stationary image segmentation using triplet Markov chains", Advanced Concepts for Intelligent Vision Systems (ACVIS 04), Aug. 31-Sept. 3, Brussels, Belgium, 2004.

[7] J. Marroquin, S. Mitter, T. Poggio, 'Probabilistic solution of ill-posed problems in computational vision," Journal of the American Statistical Association, 82, pp. 76-89, 1987.

[8] W. Pieczynski, D. Benboudjema, and P. Lanchantin, 'Statistical image segmentation using Triplet Markov Fields", SPIE's International Symposium on Remote Sensing, September 22-27, Crete, Greece, 2002.

[9] W. Pieczynski, "Statistical image segmentation", Machine Graphics and Vision, Vol. 1, No. 1/2, pp. 261268, 1992.

[10] G. Winkler, "Image analysis, random fields and Markov Chain Monte Carlo Methods: a mathematical introduction", Springer, 2003. 